\title{
Twist-three observables in deeply virtual Compton scattering on the nucleon.
}

\author{
A.V. Belitsky ${ }^{a, c}$, A. Kirchner ${ }^{b}$, D. Müller ${ }^{b, c}$, A. Schäfer ${ }^{b}$ \\ ${ }^{a}$ C.N. Yang Institute for Theoretical Physics \\ State University of New York at Stony Brook \\ NY 11794-3840, Stony Brook, USA \\ ${ }^{b}$ Institut für Theoretische Physik, Universität Regensburg \\ D-93040 Regensburg, Germany \\ ${ }^{c}$ Fachbereich Physik, Universität Wuppertal \\ D-42097 Wuppertal, Germany
}

\begin{abstract}
We study twist-three effects in deeply virtual Compton scattering on an unpolarized spin- $1 / 2$ target. A careful definition of observables as Fourier moments w.r.t. the azimuthal angle allows for a clear separation of twist-two and -three effects. Although the latter are power suppressed, they give leading contributions to the twist-three asymmetries and do not affect the twist-two observables.
\end{abstract}

Keywords: deeply virtual Compton scattering, twist-three contributions, asymmetries, generalized parton distribution

PACS numbers: 11.10.Hi, 12.38.Bx, 13.60.Fz 
1. The understanding of the hadron substructure as well as the dynamics of hadron constituents requires mutual efforts from both the theoretical and experimental side. Theoretically, we have to deduce as much information as possible from perturbative and non-perturbative QCD calculations. Until now, on the experimental side the nucleon structure has been mostly probed in inclusive processes, e.g. measurements of deep-inelastic structure functions in leptoproduction experiments, which give access to parton distribution functions. Additional information has been gained from exclusive measurements of electroweak form factors sensitive at high momentum transfer to the lowest Fock component of the hadron wave function. Since recently it became clear that far richer information can be extracted from generalized parton distributions (GPDs) [1, 20, 3], which appear e.g. in the processes of deeply virtual Compton scattering (DVCS) $\ell N \rightarrow \ell^{\prime} \gamma N^{\prime}$ and exclusive leptoproduction of mesons $\ell N \rightarrow \ell^{\prime} M N^{\prime}$. The GPDs include as limits the conventional hadron characteristics alluded to before [1, 2, 阿.

The first experimental results [4, 5, 6] show the experimental feasibility of DVCS measurements. The DVCS process contributes together with the contaminating Bethe-Heitler (BH) one to exclusive electroproduction of the real photon and requires detailed theoretical studies. Obviously, the first issue is to define appropriate observables and to determine the phase space regions that would allow for a clean interpretation of experimental measurements. As has been shown in previous studies, diverse asymmetries give an access to the interference term. Here the DVCS signal gets augmented by the Bethe-Heitler process, which provides the required handle on the GPDs

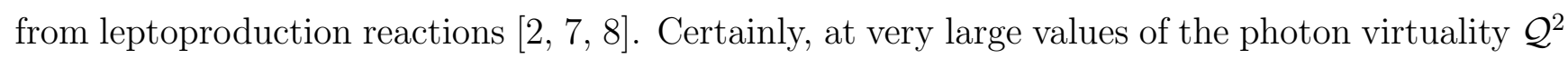
the DVCS process is dominated by its leading twist-two approximation arising from the so-called handbag diagram (including radiative corrections). Practically the onset of scaling can only be judged experimentally as present day theory does not provide necessary non-perturbative tools for the computation of soft contributions to such a reaction. For deep-inelastic scattering (DIS) it turned out, that the twist-two approximation is valid down to a rather low photon virtuality of order of a few $\mathrm{GeV}$. Although the DIS process is given by the absorptive part of forward Compton scattering, calculated by means of the same operator product expansion, the DVCS case remains an issue for studies. Since the average $\mathcal{Q}^{2}$ for all present facilities is about $4 \mathrm{GeV}^{2}$ or even below, it is vital for a clean isolation of the leading twist GPDs, to have a control over power suppressed effects.

Recently, the issues of twist-three effects [10]- [19] and target mass corrections [20] have been addressed in the literature (in the latter case only partially). In the present paper we make a step towards accomplishing the goal of handling the higher twist contributions by defining and computing twist-three observables in the DVCS cross section with polarized lepton beam and unpolarized nucleon target. 
2. The DVCS hadronic tensor is given by the time ordered product of the electromagnetic currents $j_{\mu}=\bar{\psi} \gamma_{\mu} \psi$ sandwiched between hadronic states with different momenta. In leading order of perturbation theory it reads [1]

$$
\begin{aligned}
T_{\mu \nu}(q, P, \Delta) & =i \int d x \mathrm{e}^{i x \cdot q}\left\langle P_{2}\left|T j_{\mu}(x / 2) j_{\nu}(-x / 2)\right| P_{1}\right\rangle \\
& =-\mathcal{P}_{\mu \sigma} g_{\sigma \tau} \mathcal{P}_{\tau \nu} \frac{q \cdot V_{1}}{P \cdot q}+\left(\mathcal{P}_{\mu \sigma} P_{\sigma} \mathcal{P}_{\rho \nu}+\mathcal{P}_{\mu \rho} P_{\sigma} \mathcal{P}_{\sigma \nu}\right) \frac{V_{2 \rho}}{P \cdot q}-\mathcal{P}_{\mu \sigma} i \epsilon_{\sigma \tau q \rho} \mathcal{P}_{\tau \nu} \frac{A_{1 \rho}}{P \cdot q},
\end{aligned}
$$

where we have kept all contributions to twist-three accuracy. Here we have used conventional conditions on invariants in the Bjorken limit, $-q^{2} \sim P \cdot q=$ large, $\Delta^{2}=$ small, $\xi=-q^{2} / q \cdot P=$ fixed, constructed from the vectors $P=P_{1}+P_{2}, \Delta=P_{2}-P_{1}$, and $q=\left(q_{1}+q_{2}\right) / 2$. The vectors $P_{1}$ $\left(q_{1}\right)$ and $P_{2}\left(q_{2}\right)$ refer here to the incoming and outgoing proton (photon) momentum, respectively. The reality of the final state photon implies the presence of only one scaling variable $\xi$. Current conservation in the tensor decomposition (11) is ensured here by means of the projection operator

$$
\mathcal{P}_{\mu \nu}=g_{\mu \nu}-\frac{q_{1 \mu} q_{2 \nu}}{q_{1} \cdot q_{2}} .
$$

This is consistent with an explicit calculation of the amplitude (11) to twist-three accuracy [10, 11]. In the above equation the vector $V_{2}$ is expressed in terms of the vector $V_{1 \rho}$ and the axial-vector $A_{1 \rho}$ form factors

$$
V_{2 \rho}=\xi V_{1 \rho}-\frac{\xi}{2} \frac{P_{\rho}}{P \cdot q} q \cdot V_{1}+\frac{i}{2} \frac{\epsilon_{\rho \sigma \Delta q}}{P \cdot q} A_{1 \sigma} .
$$

The latter are given as convolutions w.r.t. the momentum fraction $x, \otimes \equiv \int_{-1}^{1} d x$,

$$
V_{1 \rho}=C^{(-)}(\xi, x) \otimes v_{1 \rho}\left(x, \xi, \Delta^{2}\right), \quad A_{1 \rho}=C^{(-)}(\xi, x) \otimes a_{1 \rho}\left(x, \xi, \Delta^{2}\right)
$$

of the leading order coefficient functions

$$
\xi C_{i}^{(\mp)}(\xi, x)=Q_{i}^{2}(1-x / \xi-i \epsilon)^{-1} \mp(x \rightarrow-x),
$$

with - (+) standing for the parity even (odd) case, $Q_{i}$ being the electric charge of a quark of type- $i$, and the Fourier transforms of light-ray operators $\left(n^{2}=0\right)$ being defined by

$$
\left\{\begin{array}{c}
v_{1 \rho} \\
a_{1 \rho}
\end{array}\right\}\left(x, \xi, \Delta^{2}\right)=\int \frac{d \kappa}{2 \pi} \mathrm{e}^{i x \kappa(n \cdot P)}\left\langle P_{2}\left|\bar{\psi}(-\kappa n)\left\{\begin{array}{c}
\gamma_{\rho} \\
\gamma_{\rho} \gamma_{5}
\end{array}\right\}[-\kappa, \kappa] \psi(\kappa n)\right| P_{1}\right\rangle .
$$

The general decomposition of the vector and axial-vector amplitudes, in a complete basis of form factors to twist-three accuracy, reads

$$
\begin{aligned}
& V_{1 \rho}=P_{\rho} \frac{q \cdot h}{q \cdot P} \mathcal{H}+P_{\rho} \frac{q \cdot e}{q \cdot P} \mathcal{E}+\Delta_{\rho}^{\perp} \frac{q \cdot h}{q \cdot P} \mathcal{H}_{+}^{3}+\Delta_{\rho}^{\perp} \frac{q \cdot e}{q \cdot P} \mathcal{E}_{+}^{3}+\widetilde{\Delta}_{\rho}^{\perp} \frac{q \cdot \widetilde{h}}{q \cdot P} \widetilde{\mathcal{H}}_{-}^{3}+\widetilde{\Delta}_{\rho}^{\perp} \frac{q \cdot \widetilde{e}}{q \cdot P} \widetilde{\mathcal{E}}_{-}^{3}, \\
& A_{1 \rho}=P_{\rho} \frac{q \cdot \widetilde{h}}{q \cdot P} \widetilde{\mathcal{H}}+P_{\rho} \frac{q \cdot \widetilde{e}}{q \cdot P} \widetilde{\mathcal{E}}+\Delta_{\rho}^{\perp} \frac{q \cdot \widetilde{h}}{q \cdot P} \widetilde{\mathcal{H}}_{+}^{3}+\Delta_{\rho}^{\perp} \frac{q \cdot \widetilde{e}}{q \cdot P} \widetilde{\mathcal{E}}_{+}^{3}+\widetilde{\Delta}_{\rho}^{\perp} \frac{q \cdot h}{q \cdot P} \mathcal{H}_{-}^{3}+\widetilde{\Delta}_{\rho}^{\perp} \frac{q \cdot e}{q \cdot P} \mathcal{E}_{-}^{3},
\end{aligned}
$$


where $\Delta_{\rho}^{\perp} \equiv \Delta_{\rho}+\xi P_{\rho}, \widetilde{\Delta}_{\rho}^{\perp} \equiv i \epsilon_{\rho \Delta P q} / P \cdot q$ and the Dirac bilinears are defined conventionally by

$$
\begin{array}{ll}
h_{\rho}=\bar{U}\left(P_{2}, S_{2}\right) \gamma_{\rho} U\left(P_{1}, S_{1}\right), & e_{\rho}=\bar{U}\left(P_{2}, S_{2}\right) i \sigma_{\rho \sigma} \frac{\Delta_{\sigma}}{2 M} U\left(P_{1}, S_{1}\right), \\
\widetilde{h}_{\rho}=\bar{U}\left(P_{2}, S_{2}\right) \gamma_{\rho} \gamma_{5} U\left(P_{1}, S_{1}\right), & \widetilde{e}_{\rho}=\frac{\Delta_{\rho}}{2 M} \bar{U}\left(P_{2}, S_{2}\right) \gamma_{5} U\left(P_{1}, S_{1}\right),
\end{array}
$$

with $U$ being the nucleon bispinor.

In order to simplify our presentation, we introduce a unified convention for the twist-two $\mathcal{F} \equiv\{\mathcal{H}, \mathcal{E}, \widetilde{\mathcal{H}}, \widetilde{\mathcal{E}}\}$ and twist-three $\mathcal{F}_{ \pm}^{3} \equiv\left\{\mathcal{H}_{ \pm}^{3}, \ldots, \widetilde{\mathcal{E}}_{ \pm}^{3}\right\}$ Compton form factors. Replacing the sets of Compton form factors $\mathcal{F}$ and $\mathcal{F}_{ \pm}^{3}$ by the sets of GPDs $F_{i} \equiv\left\{H_{i}, \ldots, \widetilde{E}_{i}\right\}$ and so on, we write in analogy to Eqs. (7,8) the decomposition for the matrix elements $v_{1 \rho}$ and $a_{1 \rho}$ in terms of GPDs for quark species $i$. Here $H_{i}\left(\widetilde{H}_{i}\right)$ and $E_{i}\left(\widetilde{E}_{i}\right)$ are leading twist-two spin non-flip and spin flip GPDs, respectively, in the parity even (odd) sector. The remaining two sets, containing together eight independent functions, belong to the twist-three sector. As mentioned before, the three sets of Compton form factors are given as a convolution of the coefficient functions with GPDs, generically written as (replace $\mathcal{F} \rightarrow \mathcal{F}_{ \pm}^{3}$ and $F \rightarrow F_{ \pm}^{3}$ to get the result in the twist-three sector):

$$
\mathcal{F}\left(\xi, \Delta^{2}\right)=\sum_{i=u, d, \ldots} C_{i}^{(\mp)}(\xi, x) \otimes F_{i}\left(x, \xi, \Delta^{2}\right) .
$$

In order to deduce the GPDs from the result in Ref. [11, we decompose the (axial-) vector Dirac bilinears $h_{\rho}\left(\widetilde{h}_{\rho}\right)$ in its twist-two and -three components by means of the Dirac equation

$$
\begin{array}{r}
h_{\rho}=P_{\rho} \frac{q \cdot h}{q \cdot P}+\frac{4 M^{2}}{\left(1-\xi^{2}\right)\left(\Delta^{2}-\Delta_{\min }^{2}\right)}\left\{\Delta_{\rho}^{\perp} \xi\left(\frac{\Delta^{2}}{4 M^{2}} \frac{q \cdot h}{q \cdot P}-\frac{q \cdot e}{q \cdot P}\right)+\widetilde{\Delta}_{\rho}^{\perp}\left(\frac{\Delta^{2}}{4 M^{2}} \frac{q \cdot \tilde{h}}{q \cdot P}-\frac{q \cdot \tilde{e}}{q \cdot P}\right)\right\} \\
\widetilde{h}_{\rho}=P_{\rho} \frac{q \cdot \widetilde{h}}{q \cdot P}+\frac{4 M^{2}}{\left(1-\xi^{2}\right)\left(\Delta^{2}-\Delta_{\min }^{2}\right)}\left\{\Delta_{\rho}^{\perp} \xi\left(\left(\frac{\Delta^{2}}{4 M^{2}}-1\right) \frac{q \cdot \tilde{h}}{q \cdot P}-\frac{1}{\xi^{2}} \frac{q \cdot \tilde{e}}{q \cdot P}\right)\right. \\
\left.+\widetilde{\Delta}_{\rho}^{\perp}\left(\frac{\Delta^{2}}{4 M^{2}} \frac{q \cdot h}{q \cdot P}-\frac{q \cdot e}{q \cdot P}\right)\right\},
\end{array}
$$

where twist-four terms, proportional to $q_{\rho}$, have been neglected. The GPDs can now be easily read off from Ref. [11]. All twist-three GPDs are decomposed in the so-called Wandzura-Wilczeck terms $F_{ \pm}^{W W}$ (also calculated in Ref. [14]) and a function $F_{ \pm}^{q G q}$ that contains new dynamical information arising from antiquark-gluon-quark correlations:

$$
F_{ \pm}^{3}=F_{ \pm}^{W W}+F_{ \pm}^{q G q}
$$

According to the analysis of Ref. [11, the WW parts have the following form

$$
\begin{aligned}
& F_{+}^{W W}=\frac{1}{\xi} W_{+} \otimes \hat{d} F-\frac{1}{\xi} F-\frac{4 M^{2}}{\left(1-\xi^{2}\right)\left(\Delta^{2}-\Delta_{\min }^{2}\right)} F_{+}^{\perp}, \\
& F_{-}^{W W}=-\frac{1}{\xi} W_{-} \otimes \hat{d} F-\frac{4 M^{2}}{\left(1-\xi^{2}\right)\left(\Delta^{2}-\Delta_{\min }^{2}\right)} F_{-}^{\perp},
\end{aligned}
$$


where the convolution with the $W$-kernels

$$
W_{ \pm}\left(\frac{x}{\xi}, \frac{y}{\xi}\right)=\frac{1}{2 \xi}\left\{W\left(\frac{x}{\xi}, \frac{y}{\xi}\right) \pm W\left(-\frac{x}{\xi},-\frac{y}{\xi}\right)\right\}, \quad W(x, y)=\frac{\theta(1+x)-\theta(x-y)}{1+y},
$$

is defined as previously in Eq. (四)

$$
W \otimes \hat{d} F \equiv \int_{-1}^{1} d y W\left(\frac{x}{\xi}, \frac{y}{\xi}\right) \hat{d}(y, \xi) F(y, \xi),
$$

with the differential operator $\hat{d}(y, \xi)=y \frac{\overleftarrow{\partial}}{\partial y}-\xi \frac{\vec{\partial}}{\partial \xi}$.

Compared to the results for a (pseudo) scalar target (cf. with Eq. (24) in Ref. [12]), in the WWsector of Eq. (12) there appear in addition the functions $F_{ \pm}^{\perp}$, which arise from the decomposition of $h_{\rho}^{\perp}=h_{\rho}-P_{\rho} q \cdot h / q \cdot P$ and $\widetilde{h}_{\rho}^{\perp}=\widetilde{h}_{\rho}-P_{\rho} q \cdot \widetilde{h} / q \cdot P$, respectively. Their calculation is straightforward and they read

$$
\begin{aligned}
& H_{ \pm}^{\perp}=\mp \frac{\Delta^{2}}{4 M^{2}}\left\{\xi W_{ \pm} \otimes(H+E)-W_{\mp} \otimes \widetilde{H}\right\}, \\
& E_{ \pm}^{\perp}= \pm\left\{\xi W_{ \pm} \otimes(H+E)-W_{\mp} \otimes \widetilde{H}\right\}, \\
& \widetilde{H}_{ \pm}^{\perp}= \pm\left\{\xi\left(1-\frac{\Delta^{2}}{4 M^{2}}\right) W_{ \pm} \otimes \widetilde{H}+\frac{\Delta^{2}}{4 M^{2}} W_{\mp} \otimes(H+E)\right\}, \\
& \widetilde{E}_{ \pm}^{\perp}= \pm \frac{1}{\xi}\left\{W_{ \pm} \otimes \widetilde{H}-\xi W_{\mp} \otimes(H+E)\right\} .
\end{aligned}
$$

The antiquark-gluon-quark contributions,

$$
F_{ \pm}^{q G q}=-\int_{-1}^{1} \frac{d y}{\xi} \int_{-1}^{1} d u \frac{1-u}{2}\left\{W\left(-\frac{x}{\xi},-\frac{y}{\xi}\right) \frac{\overleftarrow{\partial^{2}}}{\partial y^{2}} S_{F}^{+}(y, u,-\xi) \pm W\left(\frac{x}{\xi}, \frac{y}{\xi}\right) \frac{\overleftarrow{\partial^{2}}}{\partial y^{2}} S_{F}^{-}(y,-u,-\xi)\right\}
$$

can be read off from the parametrization of the corresponding operators and result in eight independent functions

$$
S_{\rho}^{ \pm}=\Delta_{\rho}^{\perp} \frac{q \cdot h}{q \cdot P} S_{H}^{ \pm}+\Delta_{\rho}^{\perp} \frac{q \cdot e}{q \cdot P} S_{E}^{ \pm} \pm \widetilde{\Delta}_{\rho}^{\perp} \frac{q \cdot \widetilde{h}}{q \cdot P} S_{\widetilde{H}}^{ \pm} \pm \widetilde{\Delta}_{\rho}^{\perp} \frac{q \cdot \widetilde{e}}{q \cdot P} S_{\widetilde{E}}^{ \pm}
$$

(see Ref. [11] for details).

It turn out that only the difference $F_{+}^{3}-F_{-}^{3}$ will enter in the DVCS amplitude, thus, only four new GPDs remain at twist-three level. In the WW approximation, i.e. neglecting the quarkgluon-quark correlation, all the twist-three Compton form factors are entirely determined by the four twist-two GPDs $H, E, \widetilde{H}$ and $\widetilde{E}$.

3. In this section we define the twist-three observables, which are accessible by measuring the four-fold cross section for the process $e(k) h\left(P_{1}\right) \rightarrow e\left(k^{\prime}\right) h\left(P_{2}\right) \gamma\left(q_{2}\right)$ :

$$
\frac{d \sigma}{d x_{\mathrm{B}} d y d\left|\Delta^{2}\right| d \phi}=\frac{\alpha^{3} x_{\mathrm{B}} y}{8 \pi \mathcal{Q}^{2}}\left(1+\frac{4 M^{2} x_{\mathrm{B}}^{2}}{\mathcal{Q}^{2}}\right)^{-1 / 2}\left|\frac{\mathcal{T}}{e^{3}}\right|^{2} .
$$


This cross section depends on the Bjorken variable $x_{\mathrm{B}}=-q_{1}^{2} /\left(2 P_{1} \cdot q_{1}\right)$ (with $q_{1}=k-k^{\prime}$ and $\left.q_{1}^{2}=-\mathcal{Q}^{2}\right)$, the momentum transfer square $\Delta^{2}=\left(P_{2}-P_{1}\right)^{2}$, the lepton energy fraction $y=$ $P_{1} \cdot q_{1} / P_{1} \cdot k$, and the azimuthal angle $\phi$ between lepton and hadron scattering planes. In the twist-three approximation $\xi$ can be replaced by $x_{\mathrm{B}}$ via $\xi=x_{\mathrm{B}} /\left(2-x_{\mathrm{B}}\right)$. Furthermore, we choose a frame rotated w.r.t. the laboratory one in such a way that the virtual photon has no transverse components]. We fix our kinematics by choosing a negative $z$ component for the virtual photon momentum and a positive $x$ component of the incoming electron: $k=\left(E, E \sin \theta_{e}, 0, E \cos \theta_{e}\right)$, $q_{1}=\left(q_{1}^{0}, 0,-\left|q_{1}^{3}\right|\right), P_{1}=(M, 0,0,0)$ and $P_{2}=\left(E_{2},\left|\boldsymbol{P}_{2}\right| \cos \phi \sin \theta_{H},\left|\boldsymbol{P}_{2}\right| \sin \phi \sin \theta_{H},\left|\boldsymbol{P}_{2}\right| \cos \theta_{H}\right)$, where $\phi$ is the azimuthal angle between the lepton and hadron scattering planes.

The amplitude $\mathcal{T}$ is the sum of the virtual Compton scattering (VCS) $\mathcal{T}_{V C S}$ and the $\mathrm{BH}$ amplitude $\mathcal{T}_{B H}$. The latter one is real and is parametrized in terms of electromagnetic form factors, which we assume to be known from other measurements. The azimuthal angle dependence of each of the three terms in $\mathcal{T}^{2}=\left|\mathcal{T}_{B H}\right|^{2}+\left|\mathcal{T}_{V C S}\right|^{2}+\mathcal{I}$, where $\mathcal{I} \equiv \mathcal{T}_{V C S} \mathcal{T}_{B H}^{*}+\mathcal{T}_{V C S}^{*} \mathcal{T}_{B H}$, is given in our frame by a finite Fourier sum. In the case of an unplolarized or longitudinally polarized lepton beam, the interference term $\mathcal{I}$ and the squared DVCS amplitude $\left|\mathcal{T}_{V C S}\right|^{2}$ may be written as

$$
\begin{aligned}
& \mathcal{I}=\frac{1}{x_{\mathrm{B}} y^{3} \mathcal{P}_{1} \mathcal{P}_{2}\left(-\Delta^{2}\right)}\left\{\frac{\Delta^{2}}{\mathcal{Q}^{2}} c_{0}^{\mathcal{I}}+\sum_{m=1}^{2} K^{m}\left[c_{m}^{\mathcal{I}} \cos (m \phi)+\lambda s_{m}^{\mathcal{I}} \sin (m \phi)\right]\right\}, \\
& \left|\mathcal{T}_{\mathrm{DVCS}}\right|^{2}=\frac{1}{y^{2} \mathcal{Q}^{2}}\left\{c_{0}^{\mathrm{DVCS}}+K\left[c_{1}^{\mathrm{DVCS}} \cos (\phi)+\lambda s_{1}^{\mathrm{DVCS}} \sin (\phi)\right]\right\} .
\end{aligned}
$$

The coefficients $c_{1}^{\mathcal{I}}, s_{1}^{\mathcal{I}}$ as well as $c_{0}^{\mathrm{DVCS}}$ and $s_{0}^{\mathrm{DVCS}}$ arise at the twist-two level and their dependence on GPDs has been calculated in Refs. [7, [8]. $c_{0}^{\mathcal{I}}, c_{2}^{\mathcal{I}}, s_{2}^{\mathcal{I}}, c_{1}^{\text {DVCS }}$, and $s_{1}^{\text {DVCS }}$ provide an additional angular dependence and are given in terms of twist-two and twist-three GPDs, while the terms? that are discarded here are either proportional to $\cos (3 \phi)[\cos (2 \phi)]$ or $\sin (3 \phi)[\sin (2 \phi)]$ for the interference [squared DVCS] term. Note that all $c$ 's and $s$ 's are $\phi$ independent. There is an important difference between the interference term and the squared DVCS amplitude. The former has an additional $\phi$ dependence due to the lepton propagators]:

$$
\mathcal{Q}^{2} \mathcal{P}_{1} \equiv\left(k-q_{2}\right)^{2}=\mathcal{Q}^{2}+2 k \cdot \Delta, \quad \mathcal{Q}^{2} \mathcal{P}_{2} \equiv(k-\Delta)^{2}=-2 k \cdot \Delta+\Delta^{2},
$$

with

$$
k \cdot \Delta=-\frac{\mathcal{Q}^{2}}{2 y}\left\{1+2 K \cos \phi-\frac{\Delta^{2}}{\mathcal{Q}^{2}}\left(1-x_{\mathrm{B}}(2-y)\right)-\frac{2 M^{2}}{\mathcal{Q}^{2}}(2-y) x_{\mathrm{B}}^{2}\right\}\left\{1+\mathcal{O}\left(M^{2} / \mathcal{Q}^{2}, \Delta^{2} / \mathcal{Q}^{2}\right)\right\} .
$$

\footnotetext{
${ }^{1}$ It is a reference system related to the centre-of-mass system of $[7$ by a boost of the hadron in the $z$-direction.

${ }^{2}$ They are induced at twist-two level by the gluon transversity, which is perturbatively suppressed by $\alpha_{s}$ and is contaminated by twist-four contributions.

${ }^{3}$ For convenience we scale them with respect to $\mathcal{Q}^{2}$.
} 
The 1/Q $\mathcal{Q}$-power suppressed kinematical factor, appearing also in the Fourier series (17),

$$
K=\left\{-\frac{\Delta^{2}}{\mathcal{Q}^{2}}\left(1-x_{\mathrm{B}}\right)(1-y)\left(1-\frac{\Delta_{\min }^{2}}{\Delta^{2}}\right)\right\}^{1 / 2}\left\{1+\mathcal{O}\left(M^{2} / \mathcal{Q}^{2}, \Delta^{2} / \mathcal{Q}^{2}\right)\right\}
$$

vanishes at the kinematical boundary $\Delta^{2}=\Delta_{\min }^{2}$, determined by the minimal value

$$
-\Delta_{\min }^{2}=\frac{M^{2} x_{\mathrm{B}}^{2}}{1-x_{\mathrm{B}}+x_{\mathrm{B}} M^{2} / \mathcal{Q}^{2}}\left\{1+\mathcal{O}\left(M^{2} / \mathcal{Q}^{2}\right)\right\} .
$$

Since $\mathcal{I}$ and $\left|\mathcal{T}_{B H}\right|^{2}+\left|\mathcal{T}_{V C S}\right|^{2}$ are charge odd and even, respectively, those experimental facilities that possess beams of both charges can separately extract the BH-DVCS interference term and the sum of the squared BH and DVCS terms. Combining the charge asymmetry with different nucleon/lepton polarizations and azimuthal asymmetries [2, 7, 8] one can extract, in principle, the real and imaginary part of the four twist-two amplitudes $\mathcal{H}, \mathcal{E}, \widetilde{\mathcal{H}}$, and $\widetilde{\mathcal{E}}$ from the interference term [8]. Since $\mathcal{I}$ is linear in amplitudes involving GPDs, one gets access to the twist-two GPDs, which, however, are convoluted with the real or imaginary part of the coefficient functions. The precise definition of the Fourier coefficients is crucial for the interpretation of experimental results, since the $u$-channel lepton propagator gives a contribution that behaves like $\mathcal{P}_{1}=\left[1-y-K \cos \phi+\mathcal{O}\left(1 / \mathcal{Q}^{2}\right)\right] / y$. For instance, in the case of the single lepton spin asymmetry it may be possible that in some kinematical regions, i.e. large $y$ and not too small $\Delta^{2} / \mathcal{Q}^{2}$, this propagator effectively induces a $\sin (2 \phi)$ term that is canceled by a $s_{2}^{\mathcal{I}} \sin (2 \phi)$ term resulting in a (fake) $\sin \phi$ dependence of the cross section.

The procedure, outlined above, can also be used to access the twist-three contributions. For a successful separation of definite twist components, one should compensate for the strong azimuthal dependence of the lepton propagators in the $\mathrm{BH}$ amplitude. The $\cos (m \phi), \sin (m \phi)$ components are e.g. unraveled by weighting the cross section with $\mathcal{P}_{1} \mathcal{P}_{2}\{\cos (m \phi), \sin (m \phi)\}$. If this is not done, the magnitude of the twist-three effects can be judged by the distortion of the leading twist angular dependence $\{\cos (\phi), \sin (\phi)\} \mathcal{P}_{1}^{-1} \mathcal{P}_{2}^{-1}$.

In the following we only present the Fourier coefficients to twist-three accuracy for an unpolarized nucleon target. A complete analysis will be given elsewhere [21]. A straightforward computation provides the following analytical results for the squared DVCS amplitude

$$
\begin{aligned}
& c_{0, \text { unp }}^{\text {DVCS }}=2\left(2-2 y+y^{2}\right) \mathcal{C}_{\text {unp }}^{\text {DVCS }}\left(\mathcal{F}, \mathcal{F}^{*}\right), \\
& c_{1, \text { unp }}^{\text {DVCS }}=8 \frac{2-y}{2-x_{\mathrm{B}}} \operatorname{Re} \mathcal{C}_{\text {unp }}^{\text {DVCS }}\left(\mathcal{F}^{\text {eff }}, \mathcal{F}^{*}\right), \\
& s_{1, \text { unp }}^{\text {DVCS }}=8 \frac{y}{2-x_{\mathrm{B}}} \operatorname{Im} \mathcal{C}_{\text {unp }}^{\text {DVCS }}\left(\mathcal{F}^{\text {eff }}, \mathcal{F}^{*}\right) .
\end{aligned}
$$

Both twist-three coefficients $c_{1, \text { unp }}^{\text {DVCS }}$ and $s_{1, \text { unp }}^{\text {DVCS }}$ are given as interference of twist-two GPDs with an 
'effective' twist-three GPD

$$
\mathcal{F}^{\mathrm{eff}}=-2 \xi\left(\frac{1}{1+\xi} \mathcal{F}+\mathcal{F}_{+}^{3}-\mathcal{F}_{-}^{3}\right)
$$

where $\mathcal{F}_{ \pm}^{3}$ are defined in Eqs. (12 16). Surprisingly they have the same functional dependence as the leading twist-two function [8]:

$$
\begin{aligned}
\mathcal{C}_{\text {unp }}^{\text {DVCS }}\left(\mathcal{F}, \mathcal{F}^{*}\right)=\frac{1}{\left(2-x_{\mathrm{B}}\right)^{2}}\{ & 4\left(1-x_{\mathrm{B}}\right)\left(\mathcal{H} \mathcal{H}^{*}+\widetilde{\mathcal{H}} \widetilde{\mathcal{H}}^{*}\right)-x_{\mathrm{B}}^{2}\left(\mathcal{H} \mathcal{E}^{*}+\mathcal{E} \mathcal{H}^{*}+\widetilde{\mathcal{H}} \widetilde{\mathcal{E}}^{*}+\widetilde{\mathcal{E}} \widetilde{\mathcal{H}}^{*}\right) \\
& \left.-\left(x_{\mathrm{B}}^{2}+\left(2-x_{\mathrm{B}}\right)^{2} \frac{\Delta^{2}}{4 M^{2}}\right) \mathcal{E} \mathcal{E}^{*}-x_{\mathrm{B}}^{2} \frac{\Delta^{2}}{4 M^{2}} \widetilde{\mathcal{E}} \widetilde{\mathcal{E}}^{*}\right\} .
\end{aligned}
$$

For the interference term we found the same property for the $\cos (2 \phi) / \sin (2 \phi)$ coefficients:

$$
\begin{aligned}
c_{0, \text { unp }}^{\mathcal{I}} & =-8(2-y)\left\{\left(2-x_{\mathrm{B}}\right)(1-y)-\left(1-x_{\mathrm{B}}\right)(2-y)^{2}\left(1-\frac{\Delta_{\text {min }}^{2}}{\Delta^{2}}\right)\right\} \operatorname{Re} \mathcal{C}_{\text {unp }}^{\mathcal{I}}(\mathcal{F}) \\
& +8(2-y)(1-y) x_{\mathrm{B}}\left(F_{1}+F_{2}\right) \operatorname{Re}\left\{\frac{x_{\mathrm{B}}}{2-x_{\mathrm{B}}}(\mathcal{H}+\mathcal{E})+\widetilde{\mathcal{H}}\right\}, \\
c_{1, \text { unp }}^{\mathcal{I}} & =-8\left(2-2 y+y^{2}\right) \operatorname{Re} \mathcal{C}_{\text {unp }}^{\mathcal{I}}(\mathcal{F}), \quad s_{1, \text { unp }}^{\mathcal{I}}=8 y(2-y) \operatorname{Im} \mathcal{C}_{\text {unp }}^{\mathcal{I}}(\mathcal{F}), \\
c_{2, \text { unp }}^{\mathcal{I}} & =-16 \frac{2-y}{2-x_{\mathrm{B}}} \operatorname{Re} \mathcal{C}_{\text {unp }}^{\mathcal{I}}\left(\mathcal{F}^{\text {eff }}\right), \quad s_{2, \text { unp }}^{\mathcal{I}}=16 \frac{y}{2-x_{\mathrm{B}}} \operatorname{Im} \mathcal{C}_{\text {unp }}^{\mathcal{I}}\left(\mathcal{F}^{\text {eff }}\right) .
\end{aligned}
$$

Here the twist-two function [8]

$$
\mathcal{C}_{\text {unp }}^{\mathcal{I}}(\mathcal{F})=F_{1} \mathcal{H}+\frac{x_{\mathrm{B}}}{2-x_{\mathrm{B}}}\left(F_{1}+F_{2}\right) \widetilde{\mathcal{H}}-\frac{\Delta^{2}}{4 M^{2}} F_{2} \mathcal{E}
$$

also depends on the Dirac and Pauli form factors $F_{1}$ and $F_{2}$, respectively.

Note that twist-three GPDs, having generical discontinuities at $|x|=\xi$, enter in the Compton form factors in a singularity free combination just as for a (pseudo) scalar target. The convolutionsf of the coefficient functions $C_{i}^{\mp}$ with the $W$-kernels make this property transparent:

$$
\begin{aligned}
\mathcal{F}^{\mathrm{eff}}(\xi)= & \frac{2}{1+\xi} \mathcal{F}+2 \xi \sum_{i=u, d, \ldots}\left\{\frac{\partial}{\partial \xi} C_{i}^{3(\mp)}(\xi, x) \otimes F_{i}(x, \xi)+\frac{4 M^{2}}{\left(1-\xi^{2}\right)\left(\Delta^{2}-\Delta_{\min }^{2}\right)} \mathcal{F}_{i}^{\perp}(\xi)\right. \\
& \left.-\int_{-1}^{1} d u \frac{1+u}{\xi+x} \ln \left(\frac{2 \xi}{\xi-x-i 0}\right) \frac{\overleftarrow{\partial^{2}}}{\partial x^{2}} \otimes\left(S_{F_{i}}^{+}(-x,-u,-\xi)-S_{F_{i}}^{-}(x, u,-\xi)\right)\right\}
\end{aligned}
$$

where we have used a new convention for the coefficient function

$$
C_{i}^{3(\mp)}(\xi, x)=\frac{Q_{i}^{2}}{\xi+x} \ln \frac{2 \xi}{\xi-x-i 0} \mp\{x \rightarrow-x\},
$$

\footnotetext{
${ }^{4}$ This can be done by means of $\int_{-1}^{1} \frac{d x}{|\eta|} \frac{1}{\xi-x-i 0} W\left(\frac{x}{\eta}, \frac{y}{\eta}\right)=\frac{\operatorname{sign}(\eta)}{\eta+y} \ln \left(\frac{\eta+\xi}{\xi-y-i 0}\right)$ and proper symmetrization.
} 
with the $-(+)$ sign standing for $\mathcal{F}=\{\mathcal{H}(\widetilde{\mathcal{H}}), \mathcal{E}(\widetilde{\mathcal{E}})\}$ and

$$
\begin{aligned}
\mathcal{H}_{i}^{\perp} & =-\frac{\Delta^{2}}{4 M^{2}}\left\{\xi C_{i}^{3(-)} \otimes\left(H_{i}+E_{i}\right)-C_{i}^{3(+)} \otimes \widetilde{H}_{i}\right\}, \\
\mathcal{E}_{i}^{\perp} & =\xi C_{i}^{3(-)} \otimes\left(H_{i}+E_{i}\right)-C_{i}^{3(+)} \otimes \widetilde{H}_{i}, \\
\widetilde{\mathcal{H}}_{i}^{\perp} & =\xi\left(1-\frac{\Delta^{2}}{4 M^{2}}\right) C_{i}^{3(+)} \otimes \widetilde{H}_{i}+\frac{\Delta^{2}}{4 M^{2}} C_{i}^{3(-)} \otimes\left(H_{i}+E_{i}\right), \\
\widetilde{\mathcal{E}}_{i}^{\perp} & =\frac{1}{\xi}\left\{C_{i}^{3(+)} \otimes \widetilde{H}_{i}-\xi C_{i}^{3(-)} \otimes\left(H_{i}+E_{i}\right)\right\} .
\end{aligned}
$$

We should note that the kinematical factor in front of $\mathcal{F}^{\perp}$ drops out in the final results (20,23) and, therefore, the $1-\Delta_{\min }^{2} / \Delta^{2}$ dependence in the Fourier series (17) will not be altered. More precisely, the $\mathcal{F}^{\perp}$ dependence in the 'effective' twist-three amplitude (25) generates the following terms:

$$
\begin{aligned}
\mathcal{C}_{\text {unp }}^{\mathcal{I}}\left(\mathcal{F}^{\mathrm{eff}}\right) & =\mathcal{C}_{\text {unp }}^{\mathcal{I}}\left(\mathcal{F}^{\mathrm{eff}}, \mathcal{F}^{\perp}=0\right)+\frac{2 x_{\mathrm{B}}}{2-x_{\mathrm{B}}}\left(F_{1}+F_{2}\right) \sum_{i} C_{i}^{3(+)} \otimes \widetilde{H}_{i}, \\
\mathcal{C}_{\text {unp }}^{\mathrm{DVCS}}\left(\mathcal{F}^{\mathrm{eff}}, \mathcal{F}^{*}\right) & =\mathcal{C}_{\text {unp }}^{\mathrm{DVCS}}\left(\mathcal{F}^{\mathrm{eff}}, \mathcal{F}^{*} ; \mathcal{F}^{\perp}=0\right) \\
& +2 x_{\mathrm{B}}\left(\sum_{i} C_{i}^{3(-)} \otimes\left(E_{i}+H_{i}\right)\right)\left(\left(2-x_{\mathrm{B}}\right) \widetilde{\mathcal{H}}^{*}-x_{\mathrm{B}}(\mathcal{E}+\mathcal{H})^{*}\right) \\
& +2 x_{\mathrm{B}}\left(\sum_{i} C_{i}^{3(+)} \otimes \widetilde{H}_{i}\right)\left(\left(2-x_{\mathrm{B}}\right)(\mathcal{H}+\mathcal{E})^{*}-x_{\mathrm{B}}(\widetilde{\mathcal{H}}+\widetilde{\mathcal{E}})^{*}\right) .
\end{aligned}
$$

4. In this paper we have defined twist-two and twist-three observables in such a way that the twist-three contributions, suppressed by $\mathcal{O}\left(\Delta_{\perp} / \mathcal{Q}\right)$, do not alter the leading twist angular dependence. Thus, the corrections to the twist-two Fourier components are expected at twistfour level $\mathcal{O}\left(M^{2} / \mathcal{Q}^{2}, \Delta^{2} / \mathcal{Q}^{2}\right)$. Combining charge and spin asymmetries together with a careful extraction of the different Fourier coefficients allows a separate measurement of all coefficients of the interference and the squared DVCS term. In the latter case, the subtraction of the squared BH amplitude from the data is necessary. At the same time it drops out in single spin asymmetries.

For a lepton beam with definite charge, a separate measurement of the interference term is not possible. In this case the leading twist $\cos (\phi) / \sin (\phi)$ dependence in the cross section, which stems from the interference term, gets corrected by the twist-three contribution of the squared DVCS amplitude. Although such corrections are kinematically suppressed by $x_{\mathrm{B}} \frac{1-y}{y} \Delta^{2} / \mathcal{Q}^{2}$, their numerical value depends on the magnitude of the form factors $\mathcal{F}$.

Let us also mention that for a (pseudo) scalar target the Fourier coefficients fulfill certain constraints derived from general arguments [12]. They provide a basic test of the applicability of the operator product expansion at a given $\mathcal{Q}$-scale. We expect similar constraints for a spin- $1 / 2$ target, however, their derivation will require an evaluation of the squared amplitudes for longitudinally and transversely polarized targets. Of course, one can not test the dominance of leading 
contributions in this way or the magnitude of higher twist and $\alpha_{s}$ suppressed contaminations. This can be judged by comparing the measured scale dependence of the coefficients $c_{i}$ and $s_{i}$ with the ones arising from the evolution of GPDs and power suppressed contributions in a sufficiently large $\mathcal{Q}^{2}$-interval. Note that this task is complicated for experiments with fixed center-of-mass energy, since the kinematical variables $x_{\mathrm{B}}, y$ and $\mathcal{Q}$ do not form an independent basis in this setting. On the theoretical side the complete perturbative NLO corrections have been calculated while an estimate of twist-four effects still has to be done.

Extraction of the leading twist-two and -three Fourier coefficients allows to test models for GPDs and even to measure them in single spin asymmetries on the diagonal $x=\xi$. Having tested those predictions and processing to higher twist, one can study the validity of the 'WandzuraWilczek model' for twist-three functions suggested in Ref. [11]. A significant deviation from the latter would imply an essential contribution from antiquark-gluon-quark correlators.

To conclude, the full experimental exploration of the deep electroproduction of real photons with leptons of both charges and polarizations will lead to a direct confrontation of the data with theoretical predictions and will result in systematic tests of our understanding of the quark-gluon content of the nucleon via generalized parton distributions.

\section{References}

[1] D. Müller, D. Robaschik, B. Geyer, F.M. Dittes, J. Hořejši, Fortsch. Phys. 42 (1994) 101.

[2] X. Ji, J. Phys. G 24 (1998) 1181.

[3] A.V. Radyushkin, Phys. Rev. D 56 (1997) 5524.

[4] M. Amarian for HERMES Coll., Deeply virtual Compton scattering and exclusive meson production at HERMES, talk at the Workshop Skewed parton distributions and lepton-nucleon scattering, http://hermes.desy.de/workshop/TALKS/talks.html.

[5] P.R. Saull for ZEUS Coll., Prompt photon production and observation of deeply virtual Compton scattering, hep-ex/0003030;

L. Favart for H1 and ZEUS Coll., Deeply virtual Compton scattering at HERA, talk at ICHEP 2000, http://ichep2000.hep.sci.osaka-u.ac.jp/scan/0729/pa02/favart/index.htm].

[6] J.P. Chen et al. (Jefferson Lab), Deeply virtual Compton scattering at 6 GeV, PCCF-RI-0013, http://www.jlab.org/〜sabatie/dvcs/index.htm.

\footnotetext{
${ }^{5}$ This statement is only true at leading order in perturbation theory. Unfortunately, there are large pertubative corrections that require a careful interpretation of such experimental results.
} 
[7] M. Diehl, T. Gousset, B. Pire, J.P. Ralston, Phys. Lett. B 411 (1997) 193.

[8] A.V. Belitsky, D. Müller, L. Niedermeier, A. Schäfer, Nucl. Phys. B 593 (2001) 289; A.V. Belitsky, D. Müller, Phys. Lett. B 486 (2000) 369.

[9] I.V. Anikin, B. Pire, O.V. Teryaev, Phys. Rev. D 62 (2000) 071501.

[10] M. Pentinen, M.V. Polyakov, A.G. Shuvaev, M. Strikman, Phys. Lett. B 491 (2000) 96.

[11] A.V. Belitsky, D. Müller, Nucl. Phys. B 589 (2000) 611.

[12] A.V. Belitsky, D. Müller, A. Kirchner, A. Schäfer, Twist-three analysis of photon electroproduction with pion, hep-ph/0011314.

[13] N.A. Kivel, M.V. Polyakov, A. Schäfer, O.V. Teryaev, Phys. Lett. B 497 (2001) 73.

[14] N. Kivel and M.V. Polyakov, DVCS on the nucleon to the twist-3 accuracy, hep- ph/0010150.

[15] A.V. Radyushkin, C. Weiss, Phys. Lett. B 493 (2000) 332; Phys. Rev. D (in press), hepph/0010296.

[16] N. Kivel, M.V. Polyakov, M. Vanderhaeghen, DVCS on the nucleon: study of the twist-3 effects, hep-ph/0012136.

[17] I.V. Anikin, O.V. Teryaev, Wandzura-Wilczek approximation from generalized rotational invariance, hep-ph/0102209.

[18] B.E. White, Factorization in deeply virtual Compton scattering: Local OPE formalism and structure functions, hep-ph/0102121.

[19] P. Ball, M. Lazar, A note on Wandzura-Wilczek relations, hep-ph/0103080.

[20] A.V. Belitsky, D. Müller, Phys. Lett. B (2001) (in press), hep-ph/0102224.

[21] A.V. Belitsky, A. Kirchner, D. Müller, A. Schäfer, (in preparation). 